\title{
Basic psychological need satisfaction mediates the relationship between engaging leadership and work engagement: A cross-national study
}

\author{
Vivi Gusrini Rahmadani ${ }^{1,2}$ (b) | Wilmar B. Schaufeli ${ }^{1,3}$ | \\ Tatiana Y. Ivanova ${ }^{4}$ | Evgeny N. Osin ${ }^{4}$
}

${ }^{1}$ Research Unit, Occupational \& Organizational Psychology and Professional Learning, KU Leuven, Leuven, Belgium

${ }^{2}$ Faculty of Psychology, University of Sumatera Utara, Medan, Indonesia

${ }^{3}$ Department of Psychology, Utrecht University, Utrecht, The Netherlands

${ }^{4}$ International Laboratory of Positive Psychology of Personality and Motivation, National Research University Higher School of Economics, Moscow, Russia

\section{Correspondence}

Vivi Gusrini Rahmadani, Research Unit Occupational \& Organizational Psychology and Professional Learning, KU Leuven,

Dekenstraat 2, 3000-Leuven, Belgium.

Email: vivigusrini.rahmadani@kuleuven.be

\section{Funding information}

Russian Academic Excellence project, Grant/ Award Number: 5-100; Indonesia Endowment Fund for Education (LPDP) Scholarship

\begin{abstract}
The current study investigates the mediating role of basic psychological need for satisfaction at work (i.e., autonomy, relatedness, and competence) in the relationship between engaging leadership (i.e., inspiring, strengthening, empowering, and connecting) and work engagement. Also, we are proposing and testing an additional need for meaningfulness that plays a similar mediating role. Data were collected from two independent samples from Indonesia $(n=607$ state-owned company employees) and Russia ( $n=384$ civil servants). Results of both samples confirmed that basic psychological need satisfaction (autonomy, relatedness, competence, and meaningfulness) mediated the relationship between engaging leadership and work engagement. Multigroup analysis revealed that the parameters of the mediation model were invariant across both national samples, supporting the cross-national validity of the model. When the mediating role of the satisfaction of the need for meaningfulness was tested separately, this appeared only the case in the Russian and not in the Indonesian sample.
\end{abstract}

\section{KEYWORDS}

basic psychological need satisfaction, cross-national validity, engaging leadership, work engagement 


\section{1 | INTRODUCTION}

Research and practices of work engagement are growing as it has a positive impact on employees as well as organizations (Schaufeli, 2012). The most widely used scholarly definition of work engagement describes it as "a positive, fulfilling, work-related state of mind that is characterized by vigor, dedication, and absorption (Schaufeli, Salanova, Gonzalez-Roma, \& Bakker, 2002; p. 74)." The vigor component refers to high levels of energy and perseverance, the dedication component refers to a sense of significance, inspiration, and involvement and the absorption component refers to being focused, fully concentrated, and attentive to one's work. By employing engaged workers, organizations may increase not only the performance at the individual and team level, but also at the organization and business unit level (Salanova, Rodríguez-Sánchez, Schaufeli, \& Cifre, 2014; Schneider, Marcey, \& Barbera, 2009; Schneider, Yost, Kropp, Kind, \& Lam, 2018; Torrente, Salanova, Llorens, \& Schaufeli, 2012). Thus, employees with high levels of work engagement constitute the organizations' most valuable competitive advantage (Schaufeli, 2012).

Despite the importance of work engagement, the prevalence of highly engaged workers in organizations worldwide seems to decline (Czarnowsky, 2008), which necessitates human resource development (HRD) scholars and practitioners to develop research agendas and practical strategies to nurture engaged workers (Shuck, Rocco, \& Albornoz, 2011). Previous studies emphasized the role of the leaders in increasing employees' work engagement (Breevaart, Bakker, Demerouti, \& van den Heuvel, 2015; Tims, Bakker, \& Xanthopoulou, 2011). Leaders are important agents to nurture and manage work engagement among their employees. A recent meta-analysis found that various leadership styles are positively related to work engagement, such as ethical leadership ( $k=9 ; \rho=.58$ ), transformational leadership $(k=36 ; \rho=.46)$, servant leadership $(k=3 ; \rho=.43)$, authentic leadership $(k=17 ; \rho=.38)$, and empowering leadership ( $k=4 ; \rho=.35$ ) (DeCuypere $\&$ Schaufeli, 2018). Although these leadership styles are related to work engagement, in-depth knowledge about the underlying mechanism is still lacking. It remains unclear how certain leadership styles may increase work engagement. This has-at least partly-to do with the lacking theoretical foundation of current leadership concepts such as transformational leadership (van Knippenberg \& Sitkin, 2013). Thus, there is a need for developing an alternative, theory-based, specific leadership conceptualization that may be inherently linked to work engagement.

The current study attempts to address this need by proposing and testing the novel concept of engaging leadership, which was developed by Schaufeli (2015) and was firmly rooted in self determination theory (SDT; Deci \& Ryan, 2000). Based on the systematic review by Bormann and Rowold (2018) on construct proliferation in leadership style research, it can be argued that engaging leadership differs from other existing leadership concepts. First, while transformational leadership is categorized as a change-oriented leadership style, servant leadership, authentic leadership, and ethical leadership are categorized as relations-oriented leadership styles (cf., Yukl, Gordon, \& Taber, 2002). Contrary to transformational leadership, engaging leadership is relations-oriented because engaging leaders are supportive and promote their followers' well-being. Second, Bormann and Rowold (2018) posited that the core of "narrow" leadership constructs "bases on a single pillar" (p. 163), and they predict narrow outcomes, such as ethical leadership. Clearly, the concept of engaging leadership is narrow because it focuses on leadership behaviors that foster work engagement, which differs from broad leadership constructs, such as transformational or transactional leadership.

The main difference between engaging leadership and the other leadership concepts is, however, that the former is firmly rooted in a well-established theory, whereas previous leadership concepts are criticized because they lack a detailed theoretical description of the underlying processes (Bormann \& Rowold, 2018), most notably transformational leadership (van Knippenberg \& Sitkin, 2013). Moreover, Bormann and Rowold (2018) suggested that leadership concepts might use SDT because this motivational theory allows a more parsimonious description of the mechanisms underlying leadership behaviors. In line with this suggestion, the engaging leadership concept explicitly builds on that theory (SDT), and therefore, constitutes a positive exception, for instance, compared to authentic leadership or ethical leadership. In sum, even though each leadership concept is-to some extent-related to any other, engaging leadership can be seen as a distinct (narrow), relationship-oriented, and theory-based leadership concept. 
Tellingly, Bormann and Rowold (2018, p. 162) concluded that a one-sided critical stance regarding newer leadership styles in terms of proliferation is premature (e.g., because high correlations are likely to result from method bias).

The concept of engaging leadership was developed by Schaufeli (2015) and SDT (Deci \& Ryan, 2000) was chosen as the theoretical foundation because research showed that the fulfillment of basic psychological needs at work, as stipulated by SDT, is positively related to work engagement (Schreurs, van Emmerik, Van den Broeck, \& Guenter, 2014; Sulea, van Beek, Sarbescu, Virga, \& Schaufeli, 2015; Van den Broeck, Vansteenkiste, De Witte, \& Lens, 2008). When employees' basic needs for competence, relatedness, and autonomy are satisfied they are more likely to be engaged at work, that is. Engaging leaders might foster the fulfillment of these basic needs by strengthening, connecting, empowering, and inspiring employees, respectively, and hence increase their followers' levels of work engagement (Schaufeli, 2015). In addition to these three original basic needs, employee's need for meaningfulness was included in this study as well. It is expected that engaging leader behaviors are positively associated with work engagement through the fulfillment of followers' basic needs for autonomy, competence, relatedness, and meaningfulness.

The mediating role of basic psychological need satisfaction is studied in a cross-national setting, more particularly in Indonesia and Russia. Conveniently selected, Indonesia and Russia differ in terms of their socio-demography, economy, and culture. Indonesia is an Asian, tropical, lower-middle country with a very high population density. In contrast, Russia is a colder Eurasian country with a very low population density and a Gross Domestic Product (GDP) per capita that is three times higher than Indonesia. Also, both countries differ in culture, as illustrated by the cultural dimension scores of Hofstede (2001). That is, compared to Russia, Indonesian culture is characterized by less power distance (78 vs. 93 ), individualism (14 vs. 39), uncertainty avoidance (48 vs. 95 ), and long-term orientation (62 vs. 81 ), and more by masculinity ( $46 \mathrm{vs.} 36$ ) and indulgence ( $38 \mathrm{vs.} 20$ ). The purpose of the current study is not to investigate cross-national differences per se, but rather to test the invariance of the hypothesized mediation model across two countries that differ in many respects.

In sum, the added value of this study is to contribute to the validity of the engaging leadership concept as a novel specific leadership style by showing its impact on employee's work engagement through the satisfaction of their basic psychological needs (mediation model). A special feature is the incorporation of an additional need for meaningfulness, whose mediating role will be tested separately. Moreover, the robustness of the mediation models will be investigated in two samples simultaneously that originate from countries that differ socially, economically, and culturally.

\section{2 | THE JOB-DEMANDS RESOURCES MODEL AND LEADERSHIP}

Most studies on work engagement use the Job-Demands Resources (JD-R) model (Bailey, Madden, Alfes, \& Fletcher, 2017), whereas other theories are Emotional Contagion Theory (Bakker, Westman, \& Schaufeli, 2007), SDT (Van den Broeck et al., 2008), Conservations of Resource Theory (Xanthopoulou, Bakker, Demerouti, \& Schaufeli, 2009), Social Cognitive Theory (Salanova, Llorens, \& Schaufeli, 2011), and Broaden and Build Theory (Ouweneel, Le Blanc, \& Schaufeli, 2011). However, the JD-R model has been criticized of being a descriptive rather than an explanatory framework, meaning that other psychological theories should be integrated into the model to explain and understand the underlying processes (Schaufeli \& Taris, 2014). In the current study, we use SDT (Deci \& Ryan, 2000) for that purpose; that is, for understanding the underlying mechanism that might explain the relationship between leadership and work engagement.

According to the JD-R model, work engagement mediates between job resources and positive outcomes (wellbeing, performance), whereas in contrast, burn out mediates between job demands and negative outcomes (health problems) (Schaufeli \& Taris, 2014). The former is called the motivational process, whereas the latter is called the health impairment process. Schaufeli (2015) integrated leadership into the JD-R model, based on the principle that 
leaders are supposed to balance the job demands and job resources of their followers in such a way that they remain healthy, motivated, and productive. They do so by managing the allocation and the impact of job demands and job resources on their followers.

The current study focuses exclusively on the motivational process of the JDR-model and uses SDT as an explanatory theory on how leaders can impact employees' work engagement. Leaders have an important role in providing their followers (team members) with job resources (Chang, Hsu, Liou, \& Tsai, 2013; Demerouti, Nachreiner, Bakker, \& Schaufeli, 2001; Salanova, Llorens, Cifre, Martínez, \& Schaufeli, 2014). For example, leaders may increase employees' job resources by giving them support, autonomy, and feedback. In doing so, they will satisfy their followers' basic psychological needs, as will be argued later, and consequently increase their level of work engagement. On the other hand, leaders may increase employees' job demands by giving them a lot of workloads, tight schedule, and no support.

\section{3 | ENGAGING LEADERSHIP AND SELF DETERMINATION THEORY}

SDT is a well-known and widely empirically tested theory of human motivation and optimal functioning, which was also applied in occupational health psychology (Van den Broeck et al., 2008). According to SDT, individuals are growth-oriented agents who actively interact with their environment (E. Deci \& Ryan, 2000). However, to reach the growth and self-determined stage, people's basic needs should be satisfied, as these act as resources that nourish their growth-oriented tendency (Van den Broeck et al., 2008). According to SDT, basic psychological needs are defined as "those nutriments that must be procured by a living entity to maintain its growth, integrity, and health" (Deci \& Ryan, 2000, p. 326). Thus, the satisfaction of basic psychological needs should be given a priority, to ensure positively engaged and optimally motivated employees (Van den Broeck et al., 2008).

At work, social-contextual factors such as work climate, job design, and leadership have an impact on employees' basic need satisfaction (BNS) and hence their mental health. Moreover, in SDT, autonomy-supportive social contexts facilitate autonomous motivation or self-determined motivation, such as the manager's autonomy support by satisfying the basic needs (Gagné \& Deci, 2005). Thus, supervisor's or managerial autonomy support is identified as one of the variables that predict the employees' BNS, and in turn, increase engagement and well-being (Deci et al., 2001).

Three basic psychological needs are distinguished in SDT; the need for autonomy, relatedness, and competence. The need for autonomy is defined as the desire to experience a sense of ownership over one's behavior. The need for relatedness is defined as the desire to be part of a group and to feel connected with others. Finally, the need for competence is defined as the desire to be effective in dealing with environmental challenges and being capable of achieving desired outcomes. Although the need for meaningfulness has not been identified as a separate basic need by SDT so far, theoretical and empirical arguments have been proposed in favor of it (e.g., Andersen, Chen, \& Carter, 2000; Hadden \& Smith, 2019). Need for meaningfulness has similar characteristics to basic psychological needs, namely, it has a motivational aspect, it promotes well-being, and it is unique (Hadden \& Smith, 2019). Furthermore, based on their two diary studies, it is argued that need for meaning is a robust predictor of psychological well-being and it is uniquely correlated with well-being indicators, even in the presence of the three other basic needs (Hadden \& Smith, 2019).

However, so far, the authors of SDT view meaningfulness as an outcome of basic psychological need satisfaction rather than a need in itself (Ryan \& Deci, 2017). In contrast and following Baumeister (1991) and Frankl (1992), we believe that the need for meaningfulness, which is defined as the desire to be engaged in activities that are useful, important, significant, and are in line with one's personal values, plays a fundamental role in human motivation. Besides, meaningfulness has a strong positive association with work engagement (May, Gilson, \& Harter, 2004). The current research tests whether the need for meaningfulness can be considered a constituent element of psychological need satisfaction, which plays a mediating role between engaging leadership and employee's work engagement. 


\section{4 | ENGAGING LEADERSHIP AND WORK ENGAGEMENT}

While Gagné and Deci (2005) measured autonomy support in relation to BNS in a certain way, Schaufeli (2015) proposed four components of engaging leadership, namely, empowering, connecting, strengthening, and inspiring, which may facilitate the satisfaction of followers' basic psychological needs for autonomy, relatedness, competence, and another important need; the need for meaningfulness. The corresponding behaviors that are also rooted in SDT referred to leadership competencies focusing on encouraging autonomy, deepening relatedness, and building competence (Fowler, 2018). By empowering employees, for instance, by giving them a voice, engaging leaders satisfy their need for autonomy. By connecting employees with others in their team, engaging leaders satisfy the employees' need for relatedness. By strengthening employees, for instance, through delegating tasks and responsibilities and providing challenging jobs, engaging leaders satisfy the employees' need for competence. Finally, by inspiring employees, engaging leaders acknowledge their personal contribution to the significant overall goal of the team or organization and hence satisfy their need for meaningfulness.

As a result, the satisfaction of each basic need may increase the components of work engagement. By having a sense of autonomy, employees' self-determination is triggered, because they feel greater freedom to do their work. Hence, their work goals can be brought more in line with their personal goals and because of this congruence, work goals may be more successfully integrated into the self and thus boost a sense of pride and enthusiasm with their work (dedication). By having their need of relatedness satisfied, employees feel at ease and comfortable to express themselves in their work team and to relate to others, which contributes to a positive team spirit (dedication, absorption). In addition to that, a diary study by Mäkikangas, Bakker, and Schaufeli (2017) has found that the connecting aspect of engaging leadership also encourages the job-crafting behavior of teams. In its turn, job crafting is known to be beneficial for work engagement (Tims, Bakker, Derks, \& van Rhenen, 2013; Wang, Demerouti, \& Bakker, 2016). By having the need for competence satisfied, employees experience mastery, which motivates them to invest an extra effort in their work (vigor). By having their need for meaningfulness fulfilled, employees feel that their work is useful and important, not only for themselves but also for their colleagues, customers, the organization, and perhaps even for society as a whole. This fosters a strong identification with the job, which makes it difficult to detach from it (dedication, absorption).

The mediating role of basic psychological need satisfaction in the relation between job characteristics and wellbeing has been studied by Deci et al. (2001) in a cross-cultural context. They confirmed that managerial autonomy support predicted need satisfaction among employees in both the US and Bulgaria and need satisfaction, in its turn, predicted both task engagement and employee well-being. In a similar vein, according to Meyer, Gagné, and Parfyonova (2012), the key to moving employees toward work engagement is the fulfillment of basic psychological needs as stipulated by SDT. Furthermore, previous studies showed a significant relationship between engaging leadership and work engagement through increasing job resources (Schaufeli, 2015). Hence, it can be argued that job resources are instrumental in satisfying basic psychological needs, which then foster work engagement (Deci et al., 2001).

Van den Broeck et al. (2008) reported that the satisfaction of the three basic psychological needs described in SDT partially mediated the effect of job resources on engagement (vigor). Recently, Rigby and Ryan (2018) argued that the satisfaction of employees' basic psychological needs for autonomy, competence, and relatedness leads to a higher quality of motivation for employees. Additionally, they stressed the importance of having personally meaningful goals at work for a high-quality motivation, which can be considered as being comparable to work engagement. Finally, based on a cross-lagged study, it was found that engaging leaders are essential in shaping a resourceful work context (Nikolova, Schaufeli, \& Notelaers, 2019). In sum, these findings suggest that as a consequence of engaging leadership that focuses on inspiring, strengthening, connecting, and empowering employees, basic psychological needs are fulfilled, which, in their turn, are likely to increase levels of work engagement. This leads to: 
Hypothesis 1a Basic need satisfaction (need for autonomy, competence, relatedness, and the additional need for meaningfulness) mediates the relationship between engaging leadership and work engagement.

In addition, the alternative hypothesis is tested in which the mediating role of the need for meaningfulness is investigated separately from that of the satisfaction of the three other basic needs.

Hypothesis 1b Basic need satisfaction and need for meaningfulness independently mediate the relationship between engaging leadership and work engagement.

Hypothesis 2 The proposed mediation models are invariant across both national samples originating from Indonesia and Russia.

\section{5 | METHOD}

\section{1 | Procedure}

For the Indonesian sample, the data collection started after the company officially granted permission. Conveniently selected, 700 participants, who worked at a state-owned agricultural company in Indonesia, which operates in the cultivation of palm oil and rubber, and the production, sale, and export of palm oil and rubber products, have participated. A distribution officer handed the surveys in sealed envelopes to the participants in each unit. Participants received a written description of the study along with informed consent for the survey. The surveys were completed during the working hours; participants returned the completed survey within maximum of 2 weeks in a sealed envelope to the head office collectively per unit via a distribution officer. Participation in the study was voluntary, and the participants' responses were confidential. The whole data collection process took 3 months, from April to June 2017.

The Russian sample was comprised of employees of a regional government agency overseeing and approving construction and development projects in the region and its local offices in 31 districts. The data were collected as part of an organizational survey of work conditions and workplace well-being conducted by an external research team at the request of management. The survey was completely anonymous and conducted online; the individual participants' responses were confidential. Participants received invitations to participate and informed consent forms through their corporate e-mail system. The data were collected in November and December 2016.

\section{2 | Participants}

For the Indonesian sample, participants were 607 employees; 611 returned the survey (response rate $87.3 \%$ ) but four surveys could not be used for further analyses because of missing data. All participants were males; their mean age was 44.6 years (SD = 7.7); $23.2 \%$ completed elementary education, $59.6 \%$ completed secondary education, $0.2 \%$ completed professional higher education, $16.5 \%$ completed a bachelor degree, and $0.5 \%$ completed a master degree; more than half of the participants (56.5\%) had over 20 years of job tenure.

For the Russian sample, participants were 384 employees resulting in a response rate of $60.6 \%$ (42 more participants started but did not finish the survey). The participants were mostly female (75\%) with a mean age of 40.4 years (SD 11.7); $84.1 \%$ had a bachelor or specialist degree, $14.6 \%$ had two or more degrees, and $1.3 \%$ had secondary education. Most participants had a short job tenure of $1-3$ years $(70.7 \%)$ or less than 1 year $(10.9 \%)$. 


\section{3 | Measurements}

Because all the original scales are in English, a back translation procedure was employed for all scales for both countries.

\subsection{1 | Engaging leadership}

The 12-item Engaging Leadership scale (Schaufeli, 2015) assesses the four core dimensions of engaging leadership, namely strengthening, connecting, empowering, and inspiring with three items each. Each item of strengthening (e.g., "My supervisor delegates tasks and responsibilities to team members"), connecting (e.g., "My supervisor encourages collaboration among team members"), empowering (e.g., "My supervisor gives team members enough freedom to complete their tasks"), and inspiring (e.g., "My supervisor is able to enthuse team members with his/her plans") is rated on a five-point Likert-type scale ranging from 1 (never) to 5 (always), with higher scores indicating higher levels of supervisor's engaging leadership as perceived by their followers.

The value of Cronbach's alpha for the total scale for Indonesian sample was .86. In the Russian sample, the same Engaging Leadership Scale was used; the value of Cronbach's alpha for the total scale was .95.

\subsection{2 | Basic need satisfaction}

The 9-item Basic Psychological Need Satisfaction Scale assesses the three components of basic psychological need satisfaction, namely the need for autonomy, relatedness, and competence. The first three psychological needs are based on the adaption of Basic Need Satisfaction at Work Scale (Deci et al., 2001) by Van den Broeck et al. (2008). All needs were measured with three items each: autonomy (e.g., "If I could choose, I would do things at work differently"), competence (e.g., "I really master my tasks at my job"), and relatedness (e.g., "I often feel alone when I am with my colleagues"). Additionally, 3 item needs for meaningfulness were included (i.e., My work is full of meaning for me, personally; My work is useful for other people; With my work, I contribute to something important). All 12 items use a five-point Likert scale ranging between "Strongly disagree" (1) and "Strongly agree" (5).

In the Indonesian sample, the value of Cronbach's alpha for the total scale was .79 and .65 for the separate need for the meaningfulness scale. In the Russian sample, the value of Cronbach's alpha for the total scale was .86 and for the meaningfulness scale .73 .

\subsection{3 | Work engagement}

In both samples, work engagement was assessed with the 9-item version of the Utrecht Work Engagement Scale (UWES, Schaufeli et al., 2006). Previous studies carried out in other countries have shown that the UWES has satisfactory psychometric properties (Schaufeli, 2012). The UWES assesses the three core dimensions of work engagement, namely vigor, dedication, and absorption. Each item of vigor (e.g., "At my work, I feel bursting with energy"), dedication (e.g., "I am proud of the work that I do"), and absorption (e.g., "I get carried away when I'm working") is rated on a five-point Likert-type scale ranging from 1 (never) to 5 (always), with higher scores indicating higher levels of employees' work engagement. In the Indonesian sample, the value of Cronbach's alpha for the total scale was 87. In the Russian sample, a 1-7 point Likert scale was used; the value of Cronbach's alpha for the total scale was .92.

\section{6 | RESULTS}

\section{1 | Preliminary analysis}

A confirmatory factor analysis (CFA) was conducted to assess the measurement model consisting of five correlated latent variables: engaging leadership (a second-order factor represented by its components of strengthening, 
connecting, empowering, and inspiring, which were each represented by their three corresponding items), BNS at work (a second-order factor represented by its components of autonomy, relatedness, and competence, which were each represented by their three corresponding items), need for meaningfulness (a first-order factor represented by its three items), work engagement (a second-order factor represented by its components of vigor, dedication, and absorption, which were each represented by their three corresponding items).

Maximum likelihood estimation methods were used and the goodness-of-fit of each model was measured using absolute and relative indices. The fit of the model to the data for both samples was examined with the comparative fit index (CFI) and the root mean square error of approximation (RMSEA). Furthermore, the normed fit index (NFI); incremental fit index (IFI); and Tucker-Lewis Index (TLI) were used. Values of RMSEA close to .06 or below as an indication of good fit (Hu \& Bentler, 1999), and values of all relative fit indices greater than .90 are considered as a good fit (Hoyle, 1995).

The measurement model showed an acceptable fit with the data for both the Indonesian sample $\chi^{2}=1,189.28$, $d f=440, p<.001 ;$ RMSEA $=.053 ; \mathrm{CFI}=.90 ; \mathrm{TLI}=.88, \mathrm{IFI}=.90)$, and the Russian sample $\left(\chi^{2}=1,230.39, d f=440\right.$, $p<.001 ;$ RMSEA = .065; CFI = .91; TLI = .89, IFI = .91). However, the modification indices suggested that the fit could be improved by allowing two error terms for the items "At my work, I feel bursting with energy" and "At my job, I feel strong and vigorous," and the items "I am immersed in my work" and "I get carried away when I'm working," to correlate because of their overlapping item content. The resulting measurement model fit the data slightly better both for the Indonesian sample $\left(\chi^{2}=1,130.51, d f=438, p<.001 ; \mathrm{RMSEA}=.049 ; \mathrm{CFI}=.91 ; \mathrm{TLI}=.91\right)$ and the Russian sample $\left(\chi^{2}=1,088.33, d f=438, p<.001 ; \mathrm{RMSEA}=.059 ; \mathrm{CFI}=.93 ; \mathrm{TLI}=.91\right)$.

In the Indonesian sample, one item ("My supervisor encourages team members to use their own strengths") had a low loading on its latent factor representing "strengthening" with a coefficient of .31 ( $p<.01$ ). For a collective culture like in Indonesia, using one's own strength is not perceived as strengthening. We retained the item for theoretical reasons, though. All other items loaded highly on their respective latent factors with coefficients ranging from .57 to 82 .

One item ("My supervisor delegates to his subordinates tasks and responsibilities for completing them") had a weak loading on its latent factor representing "strengthening" in the Russian sample with a coefficient of .19 $(p<.05)$. Obviously, delegating tasks is perceived differently in Russia and not seen as a way of strengthening; however, we retained the item for theoretical reasons. All other items loaded highly on their respective latent factors with coefficients ranging from .46 to .94 . A reliable measurement model was thus obtained.

Table 1 presents the means, SDs, correlation coefficients, and Cronbach alpha's of the study variables. As expected, all variables were positively correlated.

\subsection{Testing of hypotheses}

Multiple group analysis using Structural Equation Modeling (SEM) as implemented by AMOS, a short for Analysis of MOment Structures that implements the general approach to data analysis known as structural equation modeling (SEM), analysis of covariance structures, or causal modeling (Arbuckle, 1997) was conducted to test the model in both national samples simultaneously. First, the mediation model without any constraint was tested: a model that assumes the latent variable of engaging leadership with four indicators (i.e., strengthening, connecting, empowering, and inspiring) is associated indirectly with the latent variable of work engagement with three indicators (i.e., vigor, dedication, and absorption) through the mediating latent variable of basic psychological need satisfaction with four indicators (i.e., autonomy, competence, relatedness, and meaningfulness) (Hypothesis 1a/M1a).

Next, to examine the robustness of the proposed research model (M1a) and test Hypothesis 2 about the invariance of the model across the two national samples, several multigroup analyses were carried out including both samples simultaneously. The invariance across countries of the best-fitting multigroup model was assessed by comparing the unconstrained model with its constrained counterparts. M1a, the proposed (unconstrained) model was compared with the constrained models in terms of structural paths (M2), factor loadings (M3), and both structural and paths 


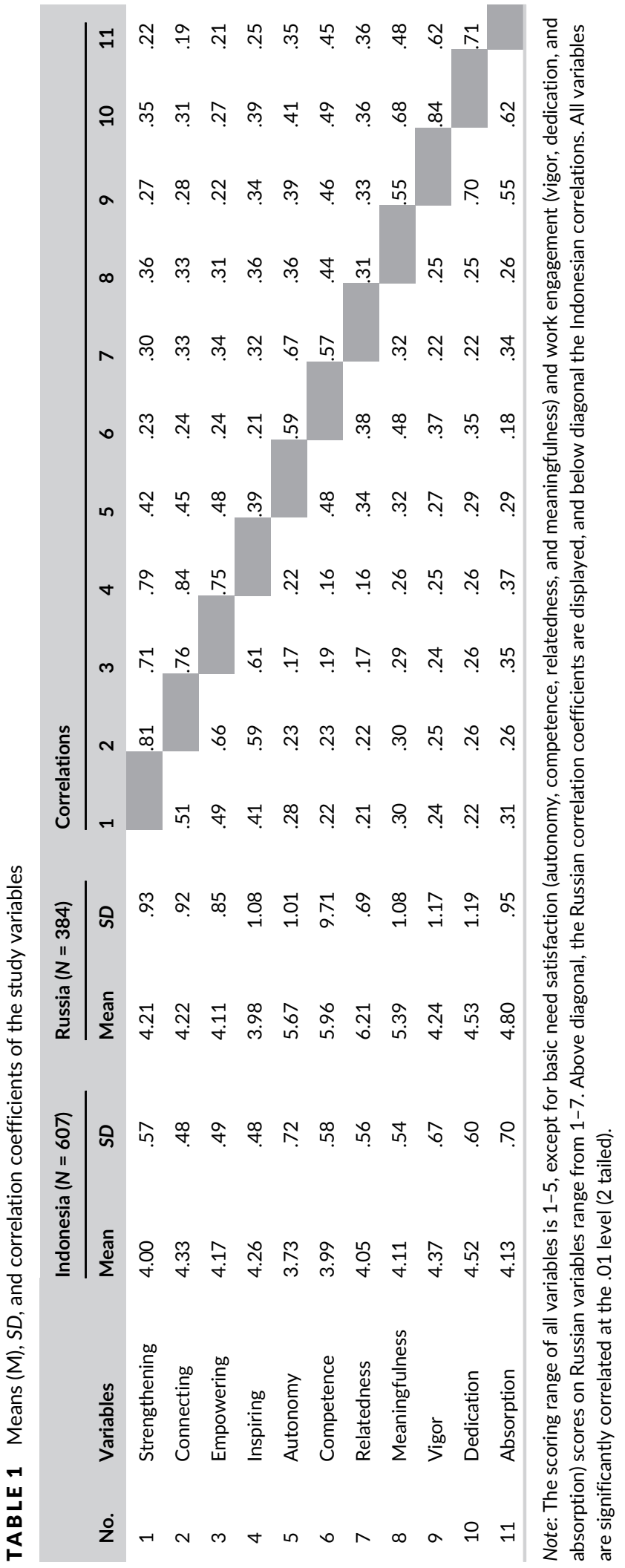


factor loadings (M4). When the fit of the constrained model to the data is not significantly worse than the fit of the unconstrained model, invariance has been demonstrated.

For the multigroup analysis of the second research model with two separate mediating latent variables (basic psychological need satisfaction and need for meaningfulness), a similar procedure was performed (Hypothesis $1 \mathrm{~b} /$ M1b). The results of the model fit related to Hypotheses $1 a-2$ and $1 b-2$ can be seen in Tables 2 and 3 , respectively.

Results, as depicted in Table 2, show that the first research model (M1a) fits the data well, with all fit indices meeting their respective criteria, and with all path-coefficients being positively significant, except the direct path of engaging leadership to work engagement for the Russian sample $(\beta=.06, p>.05)$. The model that is displayed in Figure 1 explains 34.1 and $44.3 \%$ of the variance in work engagement in the Indonesian and Russian sample, respectively. Hence, as expected, basic psychological need satisfaction mediated the relationship between engaging leadership and work engagement in both samples. In the Russian sample, basic psychological need satisfaction fully mediated this relationship, whereas in the Indonesian sample, this relationship was partially mediated. This means that Hypothesis 1a is confirmed.

Results of a series of multigroup analyses show that each competing, progressively constrained model fit the data well. Although chi-square difference tests show that constraining structural paths (M2), factor loadings (M3), and both structural paths and factor loadings (M4) to be equal across samples led to statistically significant increases of the chi-square value, the fit of these models remained similar in terms of the other fit indices. Thus, although statistically significant, the differences among the results gathered for the diverse models were actually very small and did not present systematic and meaningful variation.

Furthermore, for research model 1 hypothesis 1a, it was found that there was one insignificant direct path running from engaging leadership to work engagement in the Russian sample $(\beta=.06, p>.05)$. Additionally, we tested this engaging leadership-work engagement path in both samples using multigroup analysis by constraining this particular path. It was found that the indirect paths connecting engaging leadership and need satisfaction, and need satisfaction and work engagement, were significantly stronger in the Russian than in the Indonesian sample ( $\beta=.23$ / .06, $\Delta \chi^{2}(1)=4.29, p=.038$ ). Although in the Indonesian sample, partial mediation was observed, the direct effect of engaging leadership on work engagement was rather weak $(.23 ; p<.001)$. Next, constraining the path between

TAB LE 2 Multigroup structural equation analysis: Model comparison of research model 1

\begin{tabular}{|c|c|c|c|c|c|c|c|c|c|c|c|c|c|c|c|}
\hline \multirow[b]{2}{*}{ Model } & \multirow[b]{2}{*}{ Sample } & \multicolumn{3}{|c|}{ Structural path } & \multirow[b]{2}{*}{$x^{2}$} & \multirow[b]{2}{*}{$d f$} & \multirow[b]{2}{*}{ NFI } & \multirow[b]{2}{*}{ IFI } & \multirow[b]{2}{*}{ TLI } & \multirow[b]{2}{*}{ CFI } & \multirow[b]{2}{*}{ RMSEA } & \multirow{2}{*}{$\begin{array}{l}\text { Model } \\
\text { comparison }\end{array}$} & \multirow{2}{*}{$\Delta \chi^{2}$} & \multirow[b]{2}{*}{$d f$} & \multirow[b]{2}{*}{$p$} \\
\hline & & EL-WE & EL-BNS & BNS-WE & & & & & & & & & & & \\
\hline \multirow[t]{2}{*}{ M1a } & Indonesia & $.23 * * *$ & $.44 * * *$ & $.44 * * *$ & 39.31 & 82 & .93 & .94 & .91 & .94 & .06 & & & & \\
\hline & Russia & $.06 / \mathrm{ns}$ & $.52 * * *$ & $.63 * * *$ & & & & & & & & & & & \\
\hline \multirow[t]{2}{*}{ M2 } & Indonesia & $.12 * * *$ & $.42 * * *$ & $.51 * * *$ & 401.22 & 85 & .93 & .94 & .91 & .94 & .06 & M3-M1 & 1.91 & 3 & .012 \\
\hline & Russia & $.16 * * *$ & $.54 * * *$ & $.56 * * *$ & & & & & & & & & & & \\
\hline \multirow[t]{2}{*}{ M3 } & Indonesia & $.21 * * *$ & $.47 * * *$ & $.43 * * *$ & 494.12 & 90 & .91 & .93 & .90 & .92 & .06 & M2-M1 & 10.3 & 8 & .000 \\
\hline & Russia & $.06 / \mathrm{ns}$ & $.51 * * *$ & $.64 * * *$ & & & & & & & & & & & \\
\hline \multirow[t]{2}{*}{ M4 } & Indonesia & $.14 * * *$ & $.43 * * *$ & $.53 * * *$ & 51.99 & 93 & .91 & .92 & .89 & .92 & .06 & M4-M1 & 12.68 & 11 & .000 \\
\hline & Russia & $.09 / \mathrm{ns}$ & $.52 * * *$ & $.59 * * *$ & & & & & & & & & & & \\
\hline
\end{tabular}

M1 = Unconstrained model,

M2 = Structural paths constrained model,

M3 = Factor loadings constrained model,

M4 = Structural paths and factor loadings constrained model.

Abbreviations: BNS, basic need satisfaction; CFI, comparative fit index; $d f$, degrees of freedom; EL, engaging leadership; IFI, incremental fit index; NFI, normed fit index; RMSEA, root mean square error of approximation; TLI, Tucker-Lewis index; WE, work engagement; $\chi^{2}$, chi-square.

$* * * p<.001$. 


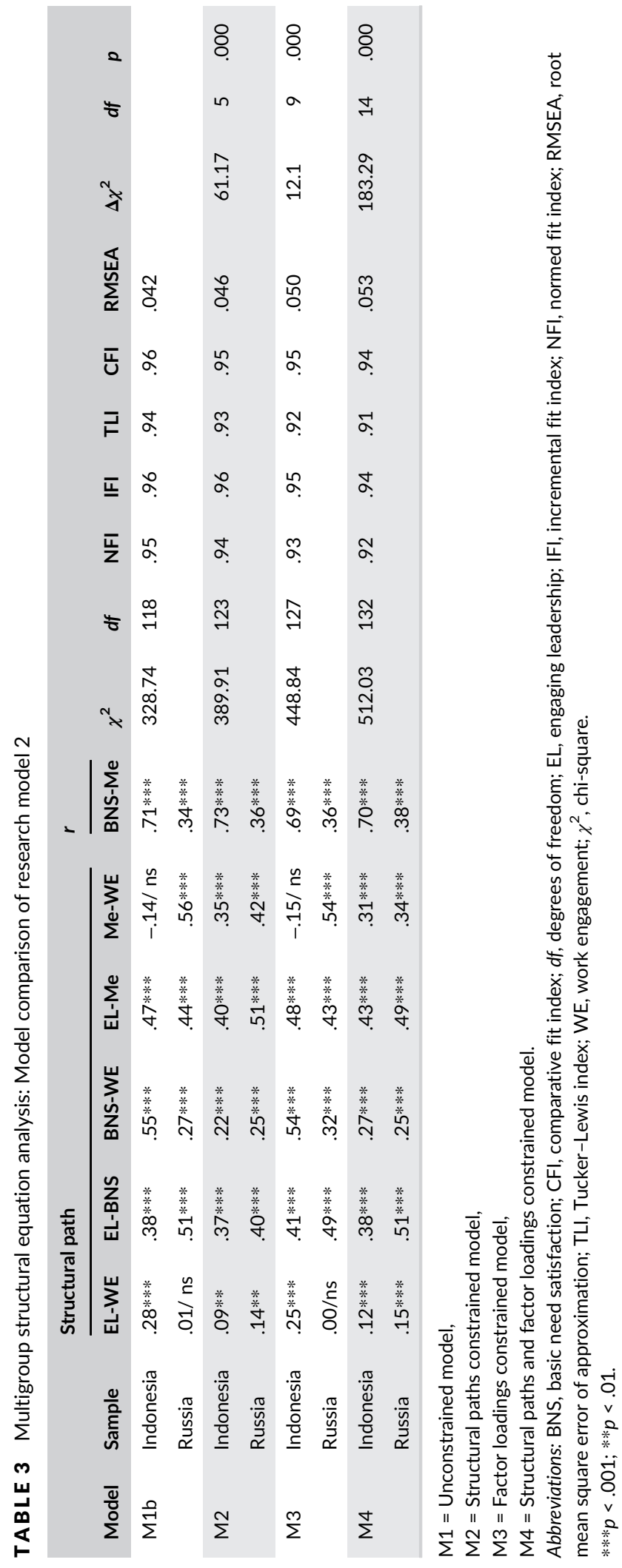




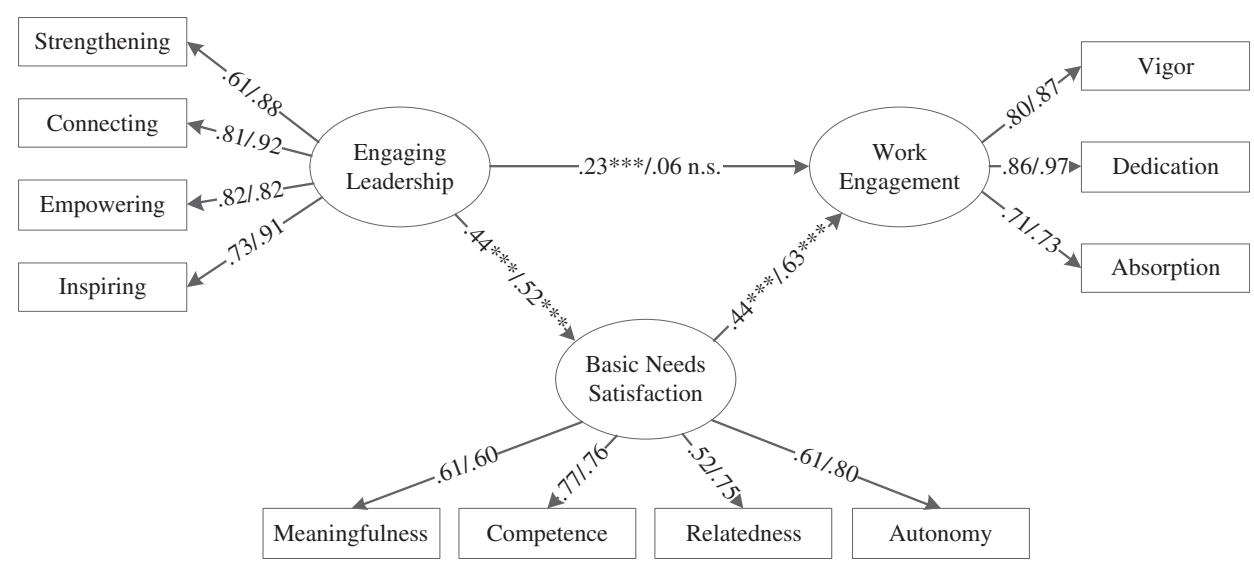

FIGURE 1 Engaging leadership and work engagement as mediated by basic psychological need satisfaction across countries (Indonesia, $n=607$ and Russia, $n=384$ ). Note: The first coefficient refers to the Indonesian samples and the second to the Russian sample

engaging leadership and BNS yielded a nonsignificant change in fit for the Indonesian and the Russian sample $\left(\beta=.44 / .52, \Delta \chi^{2}(1)=1.92, p=.16\right)$. This means this path was similar across countries. Finally, constraining the path between BNS and work engagement yielded a significant change in fit for both samples $\left(\beta=.44 / .63, \Delta \chi^{2}(1)=4.14\right.$, $p=.042$ ). This meant that the path connecting BNS and work engagement was stronger in the Russian sample.

The nonequivalent paths between the two countries suggested that other factors might moderate these paths. Hence, the first research model was supported in both countries (see the final model in Figure 1), meaning that BNS mediated the engaging leadership and work engagement relationship, even though the strength of the two of these relations was found to slightly differ in magnitude across the countries. Hence, Hypothesis 2 is supported.

The results of testing hypothesis $1 \mathrm{~b}$, as depicted in Table 3 , show that the alternative research model 2 that distinguishes between the mediation of BNS (a latent variable consisting of autonomy, relatedness, and competence) and of the need for meaningfulness also fits the data well. All fit indices meet their respective criteria, and all path coefficients are positive and significant, except the direct path of engaging leadership to work engagement in the Russian sample $(\beta=.01, p>.05)$ and the path from the need for meaningfulness to work engagement in the Indonesian sample $(\beta=-.14, p>.05)$. The model that is displayed in Figure 2 explains $36 \%$ and $54 \%$ of the variance in work engagement in the Indonesian and Russian sample, respectively.

Not surprisingly, basic psychological need satisfaction mediated again the relationship between engaging leadership and work engagement in both samples. In the Russian sample, basic psychological need satisfaction fully mediated this relationship, whereas in the Indonesian sample, this relationship was partially mediated as in the previous model. Furthermore, the need for meaningfulness acts as a significant mediator for Russian but not for Indonesian sample. This means that Hypothesis $1 \mathrm{~b}$ is partially confirmed.

For the alternative research model 2 , hypothesis $1 \mathrm{~b}$, it was also found that BNS mediated the relationship between engaging leadership and work engagement, even though, again, the strength of the relations differed slightly in magnitude across both countries. However, interestingly, this model adds new information in that the need for meaningfulness only acts as a mediator in the Russian but not in the Indonesian sample $(\beta=-.14 / .56)$. Moreover, a high correlation is observed between BNS and need for meaningfulness in the Indonesian compared to the Russian sample ( $r=.71$ versus $r=.34$ ). Thus, Hypothesis 2 is not supported (see Figure 2 ).

To summarize, in both countries the first research model fits the data well, meaning that BNS-including the need for meaningfulness-mediates the relationship between engaging leadership and work engagement, even though the strength of these relationships slightly differed across both countries. However, when the mediation of the need for 


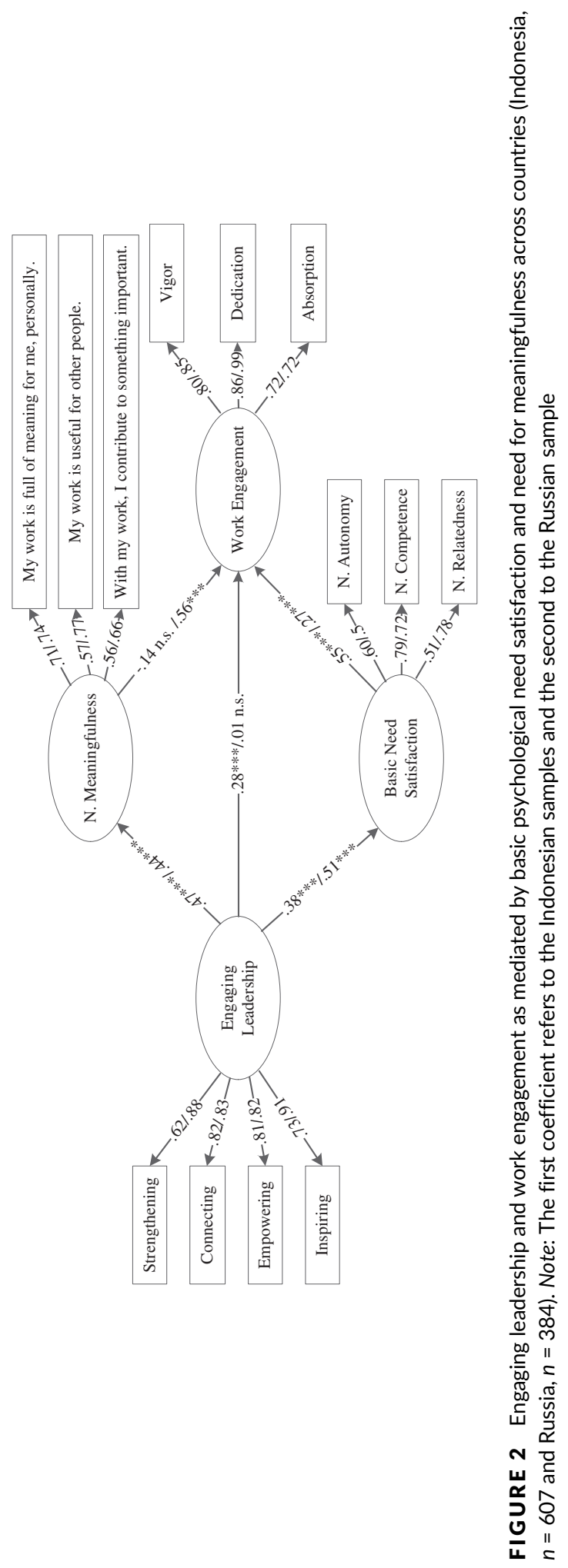


meaningfulness was tested separately, different results for both countries were observed; in the Russian sample, this mediation was confirmed, whereas in the Indonesian sample it was not.

\section{7 | DISCUSSION}

This current study investigated whether the association of engaging leadership with work engagement is mediated by basic psychological need satisfaction. By strengthening, connecting, empowering, and inspiring, engaging leaders satisfied the needs of their employees namely, the basic psychological need for autonomy, competence, and relatedness, and another additional need namely need for meaningfulness. Subsequently, BNS was, in its turn, positively related to work engagement in terms of vigor, dedication, and absorption. A series of multigroup structural equation modeling analyses provided strong evidence for the validity of the research model that assumed a mediating role of BNS in the relationship between engaging leadership and work engagement.

More specifically, we tested two different models, the first research model includes the need for meaningfulness as a constituting element of BNS. The second alternative model assumed that the need for meaningfulness acts as a separate mediator in the relationship between engaging leadership and work engagement. Across countries, engaging leadership is positively related to work engagement (for model $1 ; \beta=.23 / .06$ in Indonesia and Russia, respectively, and for model 2; $\beta=.28 / .01$ in Indonesia and Russia, respectively), and basic psychological need satisfaction (for model $1 ; \beta=.44$ / .52 in Indonesia and Russia, respectively, and for model $2 ; \beta=.38 / .51$ in Indonesia and Russia, respectively). Subsequently, basic psychological need satisfaction was positively related to work engagement (for model $1 ; \beta=.44 / .63$ in Indonesia and Russia, respectively, and for model $2 ; \beta=.55 / .27$ in Indonesia and Russia, respectively).

These findings are in line with previous studies that investigated the role of BNS at work as a mediating variable for increasing employee well-being, such as work engagement (Deci et al., 2001; Van den Broeck et al., 2008). The fact that the mediation model fits about equally well in two different countries (Indonesia and Russia) with two different occupational samples (blue-collar employees who work in production units in Indonesian state-owned company and white-collar employees who work as civil servants at a Russian government agency) attests its robustness. The cross-national validation of the model strengthens the claim that engaging leadership is associated with basic needs satisfaction, which, in its turn, is related to work engagement.

The invariant structural path connecting engaging leadership with BNS confirms the equally important role of engaging leadership for employees' need satisfaction in both countries. Additionally, results of the present study also show some small differences in the magnitude of certain paths, namely the positive relation of engaging leadership and work engagement, and the relation of BNS and work engagement. In the Russian sample, work engagement was stronger associated with BNS than was the case in the Indonesian sample. In contrast, the association of engaging leadership with work engagement was stronger in the Indonesian than in the Russian sample. These nonequivalent paths suggest that other factors might moderate these paths, such as culture, type of job, or the leader's role.

It seems that BNS acts as a mediator variable in the relationship between engaging leadership and work engagement when the three original SDT needs are involved. However, when treated as an independent mediator, the need for meaningfulness plays a different role in each country. It performs as a mediator in a similar fashion to the other SDT needs in the Russian sample, but not in the Indonesian sample. Instead, the correlation between the latent composite BNS factor and the need for meaningfulness is much stronger in the Indonesian than in the Russian sample.

One possible explanation of these differences could be that in the Indonesian sample employees occupy job positions where meaning is inherently linked with the content of the job. In the agricultural industry, the meaning and importance of one's work are obvious, namely producing foodstuff; in this case palm oil. In other words, the fulfillment of the need for meaningfulness is more or less taken for granted and, therefore, it is unlikely to have an effect on work engagement. In contrast, the Russian employees work in an administrative government agency where they complete more abstract tasks, whose meaning might not always be evident for them. In case these employees 
nevertheless find meaning in their work-and hence satisfy their need for meaningfulness-this is likely to have a positive impact on their level of work engagement. Further research is needed to confirm these speculations about the different role of meaningfulness in different occupational contexts.

To conclude, this study makes two major contributions to the literature. First of all, it confirms Schaufeli's (2015) assumption about the mediating role of BNS at work as the underlying mechanism that might explain why engaging leadership might lead to work engagement. Our study shows that this specific leadership style is related to work engagement through the satisfaction of specific psychological needs. Secondly, it appeared that an additional basic need-the need for meaningfulness-plays a similar mediating role, albeit that when this need was studied separately, mediation was only observed in the Russian and not in the Indonesian sample. Perhaps, this is due to the differences in occupation rather than the country.

\section{1 | Limitations}

This study used a cross-sectional design with self-report questionnaires. This means that no causal inferences can be made. Hence, longitudinal studies should corroborate the cross-sectional results obtained in this study. Furthermore, the use of self-report measures may lead to the occurrence of common method bias such as social desirability. For instance, the measurement of engaging leadership was based on the perception of the followers. In future studies, engaging leadership could be measured intersubjectively by using $360^{\circ}$ assessment. The same applies for work engagement (see Mazetti, Schaufeli, Guglielmi, \& Depolo, 2016).

The Indonesian and Russian samples differ in term of participant's gender; all Indonesian participants were males, whereas the majority of Russian participants were females. We used two convenience samples from organizations that operate in different cultures and sectors, which are reflected in their gender composition. It cannot be ruled out that the differences in results between the two samples are due to differences in gender distribution. However, we would like to emphasize that except for the different role of the need for meaningfulness as a separate mediator, results for both samples are rather similar.

The current study investigated engaging leadership, which is a novel construct. However, it cannot be ruled out that existing leadership concepts such as servant leadership or transformational leadership would yield similar results. Hence, in future research, the concurrent validity of engaging leadership vis-à-vis other leadership should be investigated. In fact, this agrees with Bormann and Rowold's (2018) suggestions for the introduction of a new leadership construct, which requires the establishment of its added predictive validity as well as the use of different assessment methods (e.g., self-rated behaviors and peer ratings) to avoid the danger of construct proliferation. The current study provides the first step to validate the engaging leadership construct, but it should be followed by future studies on its predictive validity vis-à-vis other leadership constructs as well as multimethod studies.

\section{2 | Practical implications}

This study confirmed that engaging leadership might increase work engagement via BNS at work. Leaders, especially supervisors and line managers, need to be aware that employees have such needs that should-at least to some extent-be fulfilled at work, and that they themselves play a role in the fulfillment of those needs. In other words, leaders in organizations need to be aware that they have a crucial role in shaping and nurturing their employees to be engaged and to foster a motivating culture.

As a first step, leaders may want to establish and promote an open and trusting team climate in which employees feel free to express their needs and preferences (Burke, Sims, Lazarre, \& Salas, 2007). This would allow leaders to assess to what extent their follower's specific psychological needs are satisfied, and discuss with them in which area this can be improved. Following Fowler's (2018) leadership competencies, which are based on SDT, organizational leaders (and HR professionals) should understand SDT and acknowledge its relevance for leadership, particularly in the one-to-one context. For example, leaders who wish to strengthen their employees have to fulfill their need for 
competence; hence they should discuss how their knowledge and skills could be increased so that they can master new, challenging tasks. Furthermore, setting employees' learning goals and support pursuing these at work will help them to grow, which will contribute to the satisfaction of their need for competence, and hence, eventually will boost their work engagement. Thus, it is important for the leaders to continuously check on their employees' level of learning and development, for instance by performing regular developmental feedback.

HRD could assist leaders by initiating programs to train and develop leaders to be engaging leaders that strengthen, connect, inspire, and empower their employees. That means that leaders at all levels should focus not only on the achievement of organizational goals but also on the fulfillment of their followers' basic needs. Preparing the skills needed to be engaging leaders is crucial, because leaders may still hesitate to change their old habit in managing employees if they do not have the skill to do so. One of the options is to develop engaging leaders by means of leadership coaching (Ely et al., 2010).

However, also, employees should be aware that their well-being at work-at least to some degree-depends on their line manager and supervisor; they should be open to the attempts of their leaders to strengthen, connect, empower, and inspire them. For example, by accepting task delegation, learning to use the new technologies, and collaborating with coworkers. In addition, employees might proactively seek satisfaction of their basic needs by, for instance, looking for challenges and opportunities for collaboration with others, and finding meaning at work; in short, by crafting their job in such a way that matches with their own needs and preferences (Bakker, 2010).

In short; it takes two to tango, both leaders and employees should collaborate and jointly build a positive, engaging work environment. This is illustrated by Shuck et al. (2011), who investigated work engagement and its implications for HRD in a large multinational service organization. Based on their empirical work, they formulated two propositions for HRD: (a) environment and person interact to create engagement or disengagement, and (b) an employee's manager plays a critical role in developing engagement. Finally, HR professionals might play a role directly by developing the potential of employees, and hence, by increasing the levels of engagement, as well as indirectly by supporting supervisors to do so.

In this study, we focused on the individual employee's unique experience of work engagement, which can be measured with a short, valid, and reliable questionnaire that can be used in the context of HRD to monitor levels of engagement of employees. Similarly, the BNS questionnaire can be used to map the level of employees' basic needs satisfaction.

\section{ACKNOWLEDGEMENTS}

This research was funded by Indonesia Endowment Fund for Education (LPDP) Scholarship. The contribution of T.Y. and E.O. was supported by the Russian Academic Excellence project "5-100."

\section{ORCID}

Vivi Gusrini Rahmadani (D) https://orcid.org/0000-0002-3726-5585

\section{REFERENCES}

Andersen, S. M., Chen, S., \& Carter, C. (2000). Fundamental human needs: Making social cognition relevant. Psychological Inquiry, 11, 269-318.

Arbuckle, J. L. (1997). AMOS user's guide version 3.6. Chicago, IL: Smallwaters Corporation.

Bailey, C., Madden, A., Alfes, K., \& Fletcher, L. (2017). The meaning, antecedents and outcomes of employee engagement: A narrative synthesis. International Journal of Management Reviews, 19(1), 31-53. https://doi.org/10.1111/ijmr.12077

Bakker, A., Westman, M., \& Schaufeli, W. (2007). Crossover of burnout: An experimental design. European Journal of Work and Organizational Psychology, 16, 220-239. https://doi.org/10.1080/13594320701218288 
Bakker, A. B. (2010). Engagement and "job crafting": Engaged employees create their own great place to work. In S. L. Albrecht (Ed.), New horizons in management. Handbook of employee engagement: Perspectives, issues, research and practice (pp. 229-244). Northampton, MA: Edward Elgar. https://doi.org/10.4337/9781849806374.00027

Baumeister, R. F. (1991). Meanings of life. New York, NY: Guilford Press.

Bormann, K. C., \& Rowold, J. (2018). Construct proliferation in leadership style research: Reviewing pro and contra arguments. Organizational Psychology Review, 8, 149-173. https://doi.org/10.1177/2041386618794821

Breevaart, K., Bakker, A. B., Demerouti, E., \& van den Heuvel, M. (2015). Leader-member exchange, work engagement, and job performance. Journal of Managerial Psychology, 30, 754-777. https://doi.org/10.1108/JMP-03-2013-0088

Burke, C. S., Sims, D. E., Lazarre, E. H., \& Salas, E. (2007). Trust in leadership: A multi-level reveiwe and integratrion. Leadership Quaterly, 18, 606-632. https://doi.org/10.1016/j.leaqua.2007.09.006

Chang, H.-T., Hsu, H. M., Liou, J. W., \& Tsai, C.-T. (2013). Psychological contracts and innovative behavior: A moderated path analysis of work engagement and job resources. Journal of Applied Social Psychology, 43, 2120-2135. https://doi. org/10.1111/jasp.12165

Czarnowsky, M. (2008). Learning's role in employee engagement: An ASTD research study. Alexandria, VA: American Society for Training \& Development.

Deci, E., \& Ryan, R. (2000). The "what" and "why" of goal pursuits: Human needs and the self-determination of behavior. Psychological Inquiry, 11, 227-268.

Deci, E. L., Ryan, R. M., Gagné, M., Leone, D. R., Usunov, J., \& Kornazheva, B. P. (2001). Need satisfaction, motivation, and well-being in the work organizations of a former eastern bloc country: A cross-cultural study of self determination. Personality and Social Psychology Bulletin, 27, 930-942.

DeCuypere, A., \& Schaufeli, W. (2018). Positive leadership styles and engagement: A meta analysis and review. Manuscript submitted for publication.

Demerouti, E., Nachreiner, F., Bakker, A. B., \& Schaufeli, W. B. (2001). The job demands-resources model of burnout. Journal of Applied Psychology, 86, 499-512.

Ely, K., Boyce, L. A., Nelson, J. K., Zaccano, S. J., Hernez-Broome, G., \& Whyman, W. (2010). Evaluating leadership coaching: A review and integrated framework. The Leadership Quarterly, 21, 585-599.

Fowler, S. (2018). Toward a new curriculum of leadership competencies: Advances in motivation science call for rethinking leadership development. Advances in Developing Human Resources, 20, 182-196. https://doi.org/10.1177/ 1523422318756644

Frankl, V. (1992). Meaning in industrial society. International Forum for Logotherapy, 15, 66-67.

Gagné, M., \& Deci, E. L. (2005). Self-determination theory and work motivation. Journal of Organizational Behavior, 26, 331-362.

Hadden, B. W., \& Smith, C. V. (2019). I gotta say, today was a good (and meaningful) day: Daily meaning in life as a potential basic psychological need. Journal of Happiness, 20, 185-202. https://doi.org/10.1007/s10902-017-9946-y

Hofstede, G. (2001). Culture's consequences: Comparing values, behaviors, institutions, and organizations across nations (2nd ed.). Thousand Oaks, CA: Sage.

Hoyle, R. H. (1995). The structural equation modeling approach: Basic concepts and fundamental issues. In R. H. Hoyle (Ed.), Structural equation modeling: Concepts, issues, and applications (pp. 1-15). Thousands Oaks, CA: Sage.

Hu, L., \& Bentler, P. (1999). Cutoff criteria for fit indices in covariance structure analysis: Conventional criteria versus new alternatives. Structural Equation Modeling, 6(1), 1-55.

Mäkikangas, A., Bakker, A. B., \& Schaufeli, W. B. (2017). Antecedents of daily team job crafting. European Journal of Work and Organizational Psychology, 26, 421-433.

May, D. R., Gilson, R. L., \& Harter, L. M. (2004). The psychological conditions of meaningfulness, safety and availability and the engagement of the human spirit at work. Journal of Occupational and Organizational Psychology, 77(1), 11-37. https://doi.org/10.1348/096317904322915892

Mazetti, G., Schaufeli, W. B., Guglielmi, D., \& Depolo, M. (2016). Overwork climate scale: Psychometric properties and relationships with working hard. Journal of Managerial Psychology, 31, 880-896. https://doi.org/10.1108/JMP-03-20140100

Meyer, J. P., Gagné, M., \& Parfyonova, N. M. (2012). Toward an evidence-based model of engagement: What we can learn from motivation and commitment research. In S. L. Albrecht (Ed.), New horizons in management. Handbook of employee engagement: Perspectives, issues, research and practice (pp. 62-73). Cheltenham: Edward Elgar. https://doi.org/10.4337/ 9781849806374.00011

Nikolova, I., Schaufeli, W., \& Notelaers, G. (2019). Engaging leader-engaged employees? A cross-lagged study on employee engagement. European Management Journal, in press. https://doi.org/10.1016/j.emj.2019.02.004

Ouweneel, E., Le Blanc, P. M., \& Schaufeli, W. B. (2011). Flourishing students: A longitudinal study on positive emotions, personal resources, and study engagement. Journal of Positive Psychology, 6, 142-153. 
Rigby, C. S., \& Ryan, R. M. (2018). Self-determination theory in human resource development: New directions and practical considerations. Advances in Developing Human Resources, 20, 133-147. https://doi.org/10.1177/1523422318756954

Ryan, R. M., \& Deci, E. L. (2017). Self-determination theory: Basic psychological needs in motivation, development, and wellness. New York, NY: The Guilford Press.

Salanova, M., Llorens, S., Cifre, E., Martínez, I., \& Schaufeli, W. (2014). Perceived collective efficacy, subjective well-being and task performance among electronic work groups: An experimental study. Small Group Research, 34(1), 43-73. https://doi.org/10.1177/1046496402239577

Salanova, M., Llorens, S., \& Schaufeli, W. B. (2011). Yes, I can, I feel good, and I just do it! On gain cycles and spirals of efficacy beliefs, affect, and engagement. Applied Psychology: An International Review, 60, 255-285.

Salanova, M., González-Romá, V., \& Bakker, A. B. (2002). The measurement of engagement and burnout: A two sample confirmatory factor analytic approach. Journal of Happiness Studies, 3(1), 71-92.

Salanova, M., Rodríguez-Sánchez, A. M., Schaufeli, W. B., \& Cifre, E. (2014). Flowing together: A longitudinal study of collective efficacy and collective flow among workgroups. The Journal of Psychology, 148, 435-455.

Schaufeli, W. B. (2012). Work engagement: What do we know and where do we go? Romanian Journal of Applied Psychology, 14(1), 3-10. https://doi.org/10.1177/0011000002301006

Schaufeli, W. B. (2015). Engaging leadership in the job demands-resources model. The Career Development International, 20, 446-463. https://doi.org/10.1108/CDI-02-2015-0025

Schaufeli, W., Bakker, A., \& Salanova, M. (2006). The measurement of work engagement with a short questionnaire: A crossnational study. Educational and Psychological Measurement, 66, 701-716.

Schaufeli, W. B., \& Taris, T. W. (2014). A critical review of the job demands-resources model: Implications for improving work and health. In G. F. Bauer \& O. Hämmig (Eds.), Bridging occupational, organizational and public health: A transdisciplinary approach (pp. 43-68). New York, NY: Springer Science+Business Media. https://doi.org/10.1007/978-94-0075640-3_4

Schneider, B., Marcey, W. H., \& Barbera, K. M. (2009). Driving customer satisfaction and financial success through employee engagement. People and Strategy, 32(2), 23-27.

Schneider, B., Yost, A. B., Kropp, A., Kind, C., \& Lam, H. (2018). Workforce engagement: What it is, what drives it, and why it matters for organizational performance. Journal of Organizational Behavior, 39, 462-480. https://doi.org/10.1002/job. 2244

Schreurs, B., van Emmerik, I. J. H., Van den Broeck, A., \& Guenter, H. (2014). Work values and engagement within teams: The mediating role of need satisfaction. Group Dynamics: Theory, Research, and Practice, 18(4), 267-281.

Shuck, B., Rocco, T., \& Albornoz, C. (2011). Exploring employee engagement from the employee perspective: Implications for HRD. Journal of European Industrial Training, 35, 300-325. https://doi.org/10.1108/03090591111128306

Sulea, C., van Beek, I., Sarbescu, P., Virga, D., \& Schaufeli, W. B. (2015). Engagement, boredom, and burnout among students : Basic need satisfaction matters more than personality traits. Learning and Individual Differences, 42, 132-138.

Tims, M., Bakker, A. B., Derks, D., \& van Rhenen, W. (2013). Job crafting at the team and individual level: Implications for work engagement and performance. Group and Organization Management, 38, 427-454. https://doi.org/10.1177/ 1059601113492421

Tims, M., Bakker, A. B., \& Xanthopoulou, D. (2011). Do transformational leaders enhance their followers' daily work engagement? Leadership Quarterly, 22, 121-131. https://doi.org/10.1016/j.leaqua.2010.12.011

Torrente, P., Salanova, M., Llorens, S., \& Schaufeli, W. B. (2012). Teams make it work: How team work engagement mediates between social resources and performance in teams. Psicothema, 24, 106-112.

Van den Broeck, A., Vansteenkiste, M., De Witte, H., \& Lens, W. (2008). Explaining the relationships between job characteristics, burnout, and engagement: The role of basic psychological need satisfaction. Work \& Stress, 22, 277-294.

van Knippenberg, D., \& Sitkin, S. B. (2013). A critical assessment of charismatic-Transformational leadership research: Back to the drawing board? The Academy of Management Annals, 7(1), 1-60. https://doi.org/1.1080/1941652.2013.759433

Wang, H., Demerouti, E., \& Bakker, A. B. (2016). A review of job crafting research: The role of leader behaviors in cultivating successful job crafters. In S. K. Parker \& U. K. Bindl (Eds.), Proactivity at work: Making things happen in organizations Series in organization and management (pp. 77-104). London, England: Taylor and Francis Ltd. https://doi.org/10.4324/ 9781315797113

Xanthopoulou, D., Bakker, A. B., Demerouti, E., \& Schaufeli, W. B. (2009). Work engagement and financial returns: A diary study on the role of job and personal resources. Journal of Occupational and Organizational Psychology, 821, $183-200$. https://doi.org/10.1348/096317908X285633

Yukl, G., Gordon, A., \& Taber, T. (2002). A hierarchical taxonomy of leadership behavior: Integrating a half century of behavior research. Journal of Leadership \& Organizational Studies, 9, 15-32. 


\section{AUTHOR BIOGRAPHIES}

Vivi Gusrini Rahmadani is a Ph.D. student at research group work, organizational and personnel psychology at KU Leuven (Belgium) and a lecturer at Faculty of Psychology at University of Sumatera Utara (Indonesia).

Wilmar B. Schaufeli is professor of work and organizational psychology at Utrecht University in the Netherlands and distinguished research professor at KU Leuven (Belgium).

Tatiana Y. Ivanova is a research fellow of the International Laboratory of Positive Psychology of Personality and Motivation at the National Research University Higher School of Economics in Moscow, Russia.

Evgeny N. Osin is currently an Associate Professor at the Department of Psychology and a Leading Research Fellow of the International Laboratory of Positive Psychology of Personality and Motivation at the National Research University Higher School of Economics in Moscow, Russia.

How to cite this article: Rahmadani VG, Schaufeli WB, Ivanova TY, Osin EN. Basic psychological need satisfaction mediates the relationship between engaging leadership and work engagement: A cross-national study. Human Resource Development Quarterly. 2019;30:453-471. https://doi.org/10.1002/hrdq.21366 